\title{
The mouse attentional-set-shifting task: A method for assaying successful cognitive aging?
}

\author{
Jared W. Young ANd Susan B. Powell \\ University of California, San Diego, La Jolla, California \\ MARK A. Geyer \\ San Diego VA Center of Excellence for Stress \& Mental Health \\ and University of California, San Diego, La Jolla, California \\ DILIP V. JESTE \\ University of California, San Diego, La Jolla, California \\ AND \\ VICTORIA B. RISBROUGH \\ San Diego VA Center of Excellence for Stress \& Mental Health \\ and University of California, San Diego, La Jolla, California
}

\begin{abstract}
Humans exhibit considerable variance in cognitive decline with age, with some exhibiting little disruption and others becoming significantly impaired. In aged rodents, individual differences in spatial memory have been used to identify putative compensatory mechanisms underlying successful hippocampal aging. However, there are few parallel rodent models of cognitive decline in frontal-cortex-mediated functions. We tested the hypothesis that, like aged humans, aged mice would exhibit greater variance in executive function measures, as compared with young mice. We examined the performance of young and aged C57BL/6N mice in the attentional-set-shifting task. Whereas young and old mice did not differ on trials-to-criterion performance, aged mice exhibited significantly greater variance in mean correct latency — selective to the extradimensional shifting stage - as compared with their younger counterparts. Thus, this task may be used to identify mechanisms underlying individual differences in decline of frontal-mediated performances with age.
\end{abstract}

The number of elderly Americans is rapidly growing, with estimates of a $300 \%$ increase in the number of adults over 60 between 2000 and 2050 (Population Division, 2001). In view of a concomitant increase in health risks with aging, research has tended to focus on ameliorating the likely impact of this trend on health care services. Just as important as treating age-related declines in health and cognitive functioning, however, is the need to understand why some people do not exhibit an age-related decline (Glatt, Chayavichitsilp, Depp, Schork, \& Jeste, 2007; Rowe \& Kahn, 1987), a phenotype sometimes termed successful aging. Although the exact phenotype of successful aging has been debated in the literature, there is a growing consensus concerning the importance of understanding the biological mechanisms of healthy aging, as well as developing early identification and putative therapies for those whose cognitive performance deteriorates with age (Glatt et al., 2007; Hendrie et al., 2006; Rowe \& Kahn, 1987; Silverman et al., 2008; Zubenko, Stiffler, Hughes, Fatigati, \& Zubenko, 2002).

Examining cognitive aging in rodents offers tight experimental control for identifying genetic, environmental, and epigenetic factors that may contribute to successful cognitive aging (cases in which cognitive performance does not deteriorate with age). Numerous tasks for rodents exist that are analogous to human cognitive tasks and show validity for selective cognitive domains (Young, Powell, Risbrough, Marston, \& Geyer, 2009). Zhang, Watson, Gallagher, and Nicolle (2007) demonstrated that, like humans, rodents exhibit variance in spatial cognitive decline with age. Age-unimpaired (AU) rats could be identified using the Morris water maze, which assessed spatial memory. Like young rats, the AU rats exhibited higher muscarinic-mediated GTP-Eu binding in the hippocampus and prefrontal cortex, as compared with age-impaired (AI) rats (Zhang et al., 2007). Cognitive reserve is one theory that proposes how older people retain cognitive performance at levels comparable to that of young subjects (Whalley, Deary, Appleton, \& Starr, 2004). Data suggest, however, that maintenance of cognitive performance with age may also be supported by adaptive behavioral strategies and/or recruitment of additional mechanisms or networks, when compared with younger subjects (Velanova, 
Lustig, Jacoby, \& Buckner, 2007). Compensatory mechanisms that may support intact spatial memory in the water maze task have been identified in aged rats. For example, young and AI rats exhibit NMDA receptor-dependent long-term depression (LTD) in the hippocampus, whereas AU rats (similar spatial memory to young) exhibited nonNMDA receptor-dependent hippocampal LTD (Lee, Min, Gallagher, \& Kirkwood, 2005). These data suggest that adaptive mechanisms may contribute to maintaining normal cognitive performance with age. Overall, these data support the use of rodents for investigating mechanisms of successful cognitive aging.

Age-associated impairment in cognitive functioning is not observed across all cognitive domains, however (Anstey \& Low, 2004; Gazzaley \& D’Esposito, 2007). Hence, when successful cognitive aging in rodents is investigated, it is important to focus on those cognitive domains that are most sensitive to age-induced impairment. One such domain with high sensitivity to age-related cognitive decline in humans is executive functioning (Gazzaley \& D'Esposito, 2007). One of the most common tests of executive function in humans is the Wisconsin card-sorting task (WCST; Eling, Derckx, \& Maes, 2008), with numerous reports on age-related decline in performance (Ashendorf \& McCaffrey, 2008; Rhodes, 2004). Performance on the WCST, however, requires contributions from working memory, reversal learning, attentional set shifting, and sustained attention, without separate measures for each of these domains (Eling et al., 2008). The complexity and multifaceted aspect of the task has led to some difficulties in the interpretation of results from this task as being relevant to any one cognitive domain (Kremen, Eisen, Tsuang, \& Lyons, 2007). In-depth analyses of age-related deficits in the WCST implicate poor shifting of an attentional set as the core deficiency in performance (Ashendorf \& McCaffrey, 2008; Rhodes, 2004). Modifications to the WCST have been made to specifically examine attentional set shifting and reversal learning, referred to as the intradimensional (ID)/extradimensional (ED) task (Owen, Roberts, Polkey, Sahakian, \& Robbins, 1991). Age-related decline in ED set shifting in this task has been reported (Owen et al., 1991). An animal analogue of the ID/ED task exists (attentional-set-shifting task [ASST]; Birrell $\&$ Brown, 2000) that exhibits high face, predictive, and construct validity for attentional set shifting in humans (Young, Powell, et al., 2009).

The ASST has been performed in rats and, more recently, has been validated in mice (Bissonette et al., 2008) and may provide a tool for assessing successful cognitive aging. Barense, Fox, and Baxter (2002) reported that aged rats had poorer performance of the ASST, as compared with young rats. Upon further examination, Nicolle and Baxter (2003) demonstrated that AI set-shifting rats exhibited lower levels of $\left[{ }^{3} \mathrm{H}\right]$ kainate binding in the cingulate cortex and higher levels of NMDA binding in the dorsomedial striatum, as compared with young and $\mathrm{AU}$ rats. Few studies have examined the genetic or epigenetic contribution to performance in this task, which may, in part, be due to the difficulty of genetic manipulation in rats, as compared with mice. One study examined ASST performance in mice overexpressing the Swedish amyloid precursor protein (APP) mutation Tg(HuAPP695.K70NM671L)2576, used as a mouse model of Alzheimer's disease, and in their wildtype litter mates 6 versus 14 months of age (Zhuo et al., 2007). Because no within-group effects of reversals or ED shifts were observed, however (Zhuo et al., 2007), it remains unclear whether the task assessed executive functioning accurately (Birrell \& Brown, 2000). Moreover, since individual differences in performance within the 14-month-old mice were not reported (Zhuo et al., 2007), it is unknown whether successful cognitive aging can be measured using this task.

We sought to examine the effects of aging (4 vs. 24 months) on ASST performance in C57BL/6 mice, an inbred strain that is commonly used in behavioral testing and is often the strain of choice when backcrossing mutants onto a particular inbred line. We hypothesized that, consistent with humans (Owen et al., 1991) and rats (Barense et al., 2002), aged C57BL/6 mice would exhibit a significant deficit in ASST performance, as compared with young mice, specific to ED shifting. Moreover, we expected that there would be a separation of performance levels within the aging group so that successful and unsuccessful cognitive-aging performers could be identified.

\section{METHOD}

Aged (24 months) male C57BL/6N mice $(n=20)$ were obtained from the NIA aged colony (Charles River). Young (5 months) C57BL/6N mice $(n=20)$ were purchased from Charles River laboratories. Training began with the mice weighing $20-40 \mathrm{~g}$. The mice were housed in pairs in a vivarium on a reversed day-night cycle (lights on at 8:00 p.m., off at 8:00 a.m.) and were maintained at 85\% of free-feeding weight with water available ad lib. Prior to training and testing, the mice were brought to the laboratory for $60 \mathrm{~min}$ between 9:00 a.m. and 6:00 p.m. All procedures were approved by the University of California, San Diego (UCSD) Institutional Animal Care and Use Committee. The UCSD animal facility meets all federal and state requirements for animal care and has been approved by the American Association for Accreditation of Laboratory Animal Care.

\section{Apparatus}

Mice readily dig in bedding placed in small bowls to retrieve a food reward, using olfactory cues to solve tasks (Young, Crawford, et al., 2007; Young, Kerr, et al., 2007; Young, Sharkey, \& Finlayson, 2009). Ceramic pots $(4.5 \times 2.5 \mathrm{~cm})$ were used for digging bowls, which were placed on platforms $(11 \times 5 \mathrm{~cm})$. Specific odors and platforms were utilized as cues to guide the selection of mice. Odors were derived from commercially available powdered spices, including ground ginger, nutmeg, garlic, coriander, thyme, and cinnamon (Albertsons). Platforms included sandpaper, wood, neoprene, metal wire, tile, and a scrubber (Homebase). Platforms were used, as opposed to a digging medium, because (1) mice do not readily manipulate their environment (Caine, Negus, \& Mello, 1999) and (2) in initial pilot studies it proved difficult to identify whether mice were manipulating the medium in order to sample the medium or to identify the correct stimulus (i.e., digging for a reward). We chose to use platforms as the second dimension, since it had previously been demonstrated that rodents can utilize platform textures to solve tasks (Stefani, Groth, \& Moghaddam, 2003; Stefani \& Moghaddam, 2003). The food reward was a single $25-\mathrm{mg}$ food pellet for each correct trial (Noyes Precision). The test apparatus was an adapted Perspex cage 
$(30 \times 18 \times 12 \mathrm{~cm})$, with clear plastic panels used to separate half of the cage into two equal sections. The two digging bowls were placed in each quarter section, with access to these sections limited by removable dividers. The removable dividers were used to deny the mice access to the other bowl after they had made a selection.

\section{Shaping}

On Day 1, the mice were introduced to the testing chamber and room and were trained to dig in unscented bedding for a food reward. On Day 2, the mice were required to dig selectively in bowls containing each of the odors they would encounter in the main task, with platforms varied so that they encountered each platform prior to testing. The criterion for a dig was defined as when the nose or paws of the mouse broke the surface of the digging medium. This criterion was used throughout testing.

\section{ASST Paradigm}

Each trial was initiated by raising the divider, allowing access to two digging bowls, one of which was baited. For the first four trials, the mice were permitted to dig in the unbaited bowl without consequence, although an error was recorded, so that if one bowl was investigated in error, the mouse could move to the second baited bowl and learn the cue contingency. Errors on subsequent trials resulted in the mouse's being denied entry to the other area, an error recorded, and the trial restarted. Trials would continue until six consecutive correct responses had been made. Throughout the test session, the mice were required to perform a series of discriminations in which they had to select a bowl on the basis of a stimulus in a particular dimension, either odor or platform. Examples of the discriminations required to be made are found in Table 1. For example, in the simple discrimination (SD), the only relevant dimension present in the example provided would be odor. Compound discrimination (CD) would introduce the second dimension (platform), but the relevant stimulus (odor) in the SD would still identify the correct bowl. The first reversal (CDR) would require the mouse to respond in the bowl with the previously irrelevant odor. Subsequently, the mice would have to perform an ID shift wherein two novel odors were presented and only one would identify the baited bowl. The second reversal (IDR) would require the mouse to respond in the previously irrelevant odor-filled bowl. The mice would then be required to perform an ED shift, wherein the previously irrelevant dimension (platform) would then be relevant and one stimulus would identify the baited bowl. The third and final reversal (EDR) would require the mice to attend to the previously irrelevant stimuli in the platform dimension. At each stage, the mice were required to make six consecutive correct responses prior to moving to the next stage. Mice were counterbalanced so that the initial SD would be either odor or platform. Stimulus combinations and locations were selected in a balanced order. Trials to criterion and errors were recorded for each stage. Given the speed-accuracy trade-off observed with increasing task difficulty in mice (Abraham et al., 2004; Rinberg, Koulakov, \& Gelperin, 2006), we also recorded mean correct latency by stage. Latencies to respond were measured using stopwatches, initiated as the doors were raised and stopped when the mouse dug in one of the choices available. The total correct latency was divided by total correct responses, to produce the mean correct latency.

\section{Statistical Analyses}

Trials to criterion, errors to criterion, and correct and incorrect response times were recorded for every mouse at each stage. Data were initially analyzed using a repeated measures three-factor ANOVA with stage (SD, CD, CDR, ID, IDR, ED, and EDR) as a within-subjects factor and with group (age) and dimension change (odor to platform, platform to odor) as between-subjects factors. Since age and stage did not significantly interact with dimension change $(F<1$, n.s.), dimension change data were not analyzed further. Different stages were also analyzed separately, because these stages are believed to be mediated by different cognitive constructs (Birrell \& Brown, 2000) and different underlying neural substrates. Thus, two-factor ANOVAs compared SD, CD, and ID as discrimination learning and CDR, IDR, and EDR as tests of reversal learning (Birrell \& Brown, 2000). Post hoc analyses for significant effects or interactions were performed using Tukey's test, with $\alpha$ set to $p<.05$. Planned $t$ test comparisons were conducted for trials to criterion and mean correct latencies on ED shifting by age, given the different cognitive constructs measured at this stage, as well as on ID shifting for comparative purposes. Where significant effects of age were observed, equality of variance was also assessed using Levene's test of equality of error variances. The relationship between ED shifting as measured by trials to criterion and correct response latencies was assessed via continuous (regression) and categorical (quartile split- top and bottom 25\%) analyses as described previously (Swerdlow et al., 2006). Data were analyzed using SPSS.

Table 1

Example of the Testing Stages and Possible Stimulus Combinations in the Attentional-Set-Shifting Task for Mice

\begin{tabular}{|c|c|c|c|c|}
\hline \multirow[b]{2}{*}{ Discrimination } & \multicolumn{2}{|c|}{ Dimension } & \multicolumn{2}{|c|}{$\begin{array}{c}\text { Exemplar } \\
\text { Combination }\end{array}$} \\
\hline & Relevant & Irrelevant & Correct & Incorrect \\
\hline Simple discrimination & odor & & $\mathrm{O} 1$ & $\mathrm{O} 2$ \\
\hline Compound discrimination (CD) & odor & platform & $\begin{array}{l}\text { O1/P1 } \\
\text { O1/P2 }\end{array}$ & $\begin{array}{l}\mathrm{O} 2 / \mathrm{P} 1 \\
\mathrm{O} 2 / \mathrm{P} 2\end{array}$ \\
\hline $\mathrm{CD}$ reversal & odor & platform & $\begin{array}{l}\text { O2/P1 } \\
\text { O2/P2 }\end{array}$ & $\begin{array}{l}\mathrm{O} 1 / \mathrm{P} 1 \\
\mathrm{O} 1 / \mathrm{P} 2\end{array}$ \\
\hline Intradimensional (ID) shift & odor & platform & $\begin{array}{l}\text { O3/P3 } \\
\text { O3/P4 }\end{array}$ & $\begin{array}{l}\mathrm{O} 4 / \mathrm{P} 3 \\
\mathrm{O} 4 / \mathrm{P} 4\end{array}$ \\
\hline ID reversal & odor & platform & $\begin{array}{l}\text { O4/P3 } \\
\text { O4/P4 }\end{array}$ & $\begin{array}{l}\mathrm{O} 3 / \mathrm{P} 3 \\
\mathrm{O} 4 / \mathrm{P} 4\end{array}$ \\
\hline Extradimensional (ED) shift & platform & odor & $\begin{array}{l}\text { P5/O5 } \\
\text { P5/O6 }\end{array}$ & $\begin{array}{l}\mathrm{P} 6 / \mathrm{O} 5 \\
\mathrm{P} 6 / \mathrm{O} 6\end{array}$ \\
\hline ED reversal & platform & odor & $\begin{array}{l}\text { P6/O5 } \\
\text { P6/O6 }\end{array}$ & $\begin{array}{l}\mathrm{P} 5 / \mathrm{O} 5 \\
\mathrm{P} 5 / \mathrm{O} 6\end{array}$ \\
\hline
\end{tabular}

Note-Mice were counterbalanced so that one half received odor as the initial relevant dimension, whereas the other half received platform. Thus, the correct exemplar is provided in bold. 


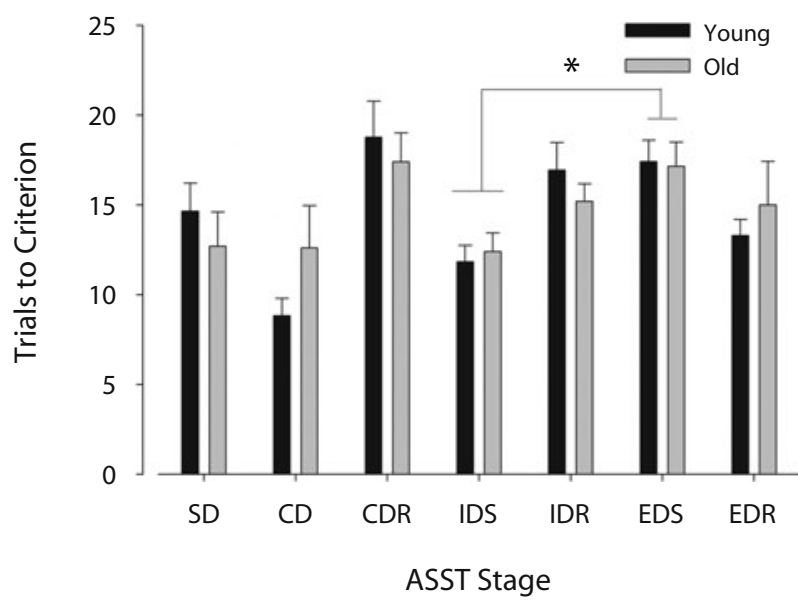

Figure 1. Trials to criterion for each stage of the attentionalset-shifting task (ASST) in young and old mice. Mice could perform the ASST when odors and platforms were used as the perceptual dimensions. The mice were required to perform six correct responses consecutively in each stage before moving on to the next stage, from simple discrimination (SD), to compound discrimination $(C D), C D$ reversal (CDR), intradimensional (ID) shift (IDS), ID reversal (IDR), extradimensional (ED) shift (EDS), and finally ED reversal (EDR). Data are presented as the mean $\pm S E M . ~{ }^{*} p<.05$.

\section{RESULTS}

\section{Overall Performance}

The performances of young and old C57BL/6N mice on the ASST were compared. Given the novelty of the parameters used, the data were analyzed for conceptual validity - that is, that performance in the ED shift was significantly worse than that in the ID shift and that this effect was apparent in young and old mice irrespective of starting stimulus dimension. A significant main effect of stage was observed on trials to criterion in the ASST $[F(6,198)=5.6, p<.001$; Figure 1]. In comparison with young mice, aged mice exhibited no significant difference in trials to criterion, with no effect of starting dimension and no stage $\times$ age interaction observed. A main effect of stage was noted on mean correct latency $[F(6,198)=$ $4.8, p<.01]$, whereas aged mice did not differ significantly from young mice in overall mean correct latency. No stage $\times$ age interaction was observed for mean correct latency. Given the differences in performance at the various stages, performance was analyzed according to the cognitive constructs represented by the stages (Birrell \& Brown, 2000).

\section{Discrimination Learning}

We examined the performance of animals in discrimination learning (stages SD, CD, and ID) because each of these required no rule shift. There were no differences in the number of trials to criterion based on age or stages, nor was there an age $\times$ stage interaction. No mean correct latency differences were observed between young and old mice or in an age $\times$ stage interaction. A main effect of stage was observed on mean correct latency, however
$[F(2,111)=5.7, p<.005]$; post hoc analyses revealed that performance at $\mathrm{CD}$ was significantly faster in all groups, as compared with the SD or ID stages $(p<.05)$.

\section{Reversal Learning}

After the discrimination stages CD, ID, and ED, the correct exemplars were reversed for CDR, IDR, and EDR, assessing reversal learning. No differences in trials to criterion were observed between young and old mice, nor was there an age $\times$ stage interaction or a main effect of stage. Young and old mice also did not differ in mean correct latency during reversal learning, nor was there an age $\times$ stage interaction.

\section{ID/ED Task Validation}

The ASST has been designed so that internal validation of attentional set formation can be assessed by comparing the performance of animals in the ID versus ED stages. Poorer ED versus ID performance would suggest that the animals had formed an attentional set to the initial stimulus dimension. Hence, we compared the ID/ED performance of all mice. When comparing overall trials to criterion, a significant ID/ED difference was observed $[F(1,70)=20.1, p<.0001$; Figure 1$]$. Tukey's post hoc analyses revealed that mice took significantly more trials to perform an ED shift, as compared with an ID shift $(p<.05)$. A significant main effect of stage was also

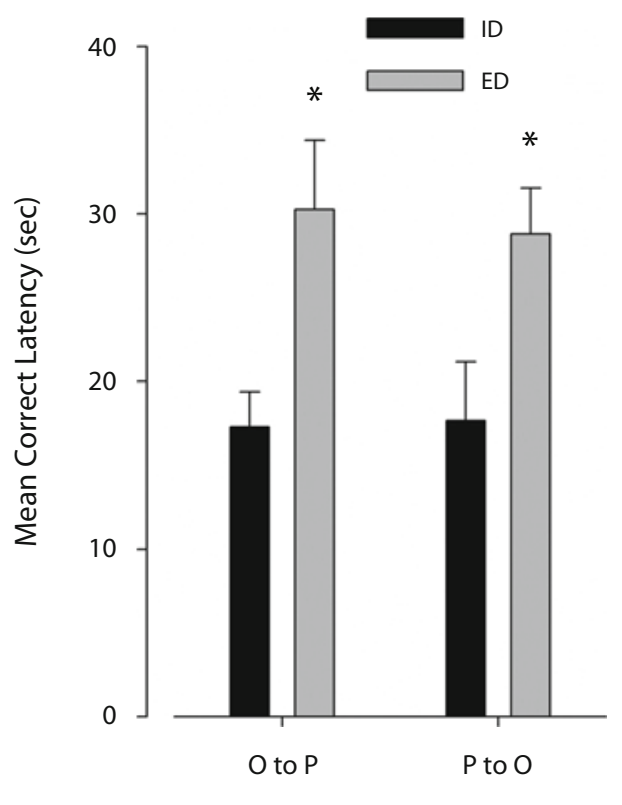

Dimensional Shift

Figure 2. Correct latency performance of mice during intradimensional (ID) and extradimensional (ED) shifts by starting dimensions, and correct latency differences for the mice when they switched from odor $(\mathrm{O})$ to platform $(\mathrm{P})$ or from $\mathrm{P}$ to $\mathrm{O}$ between ID and $E D$ shifts, respectively. Irrespective of initial starting dimension ( $O$ or $P$ ), the mice took longer to choose the correct bowls when switching to the novel stimulus dimension. Data are presented as mean $\pm S E M . \quad * p<.05$ when compared with ID shift in corresponding starting dimension. 


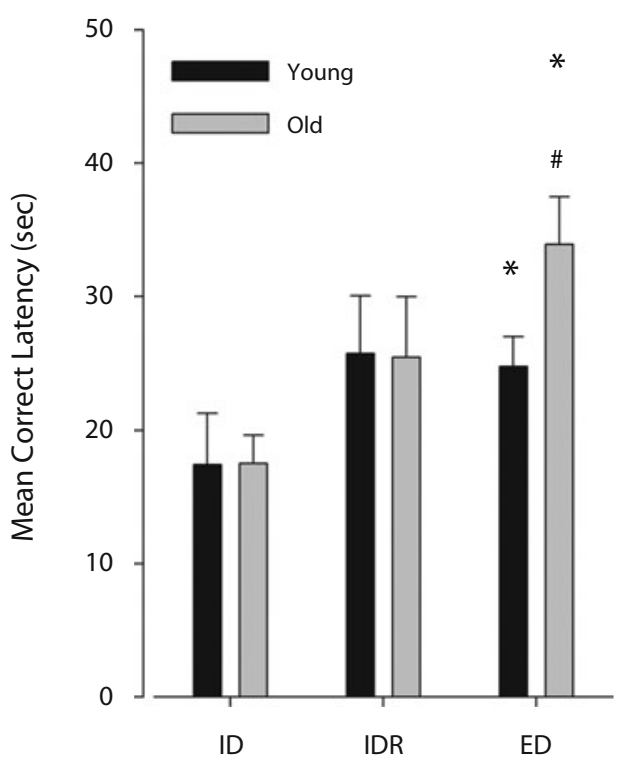

ASST Stage

Figure 3. Age-related differences in mean correct latencies for mice moving through attentional-set-shifting task (ASST) stages. Correct latencies for young and old mice were compared as they performed the intradimensional (ID) shift, ID reversal (IDR), and the extradimensional (ED) shift. Age did not affect ID or IDR correct latencies, but had negative impact on the latency for mice to complete a correct ED shift. Data are presented as mean $\pm S E M .{ }^{*} p<.05$ when compared with performance at ID shift. ${ }^{\#} p<.05$ when compared with young mice.

observed for mean correct latency $[F(1,70)=15.0, p<$ $.0001]$. Post hoc analyses revealed that mean correct latencies were longer for ED trials than for ID trials $(p<.05$; Figure 2).

\section{Aging Effects on ED Shifting}

The performances of young and old mice were compared in ID and ED shifting. These comparisons were made to determine whether there was a general aging effect on rule learning in novel stimuli (ID shifting) or whether aging selectively affected prefrontally mediated (Bissonette et al., 2008) attentional set shifting (ED shift) (Birrell \& Brown, 2000). Since ED was the only stage in which attentional set shifting was specifically examined, planned $t$ test comparisons were performed on the effect of age on ED shift performance. No effect of age on total trials to criterion was observed for ID or ED shifting. Old mice took significantly longer to make a correct response in ED shifting than did young mice, however, as measured by mean correct latency $(t=-2.2, p<.05$; Figure 3$)$. No difference in the mean correct latencies of ID shifting was observed between old and young mice $(t<1$, n.s.). Furthermore, there was no difference in the variability between young and old mice in latency to respond during ID shifting (Figure 4A). Aged mice, however, exhibited a significantly greater increase in the variability of mean correct latency during the ED shift, as compared with young mice [Levene's test of sphericity: $F(1,35)=$ 5.0, $p<.05$; Figure 4B]. These data are best represented by a scatterplot depicting the greater individual variance in mean correct latency in aged animals in the ED, but not the ID, phase of the task (Figure 4B).

Because latency changes can be interpreted as either changes in speed-accuracy trade-off or simply alterations in locomotor activity in aged mice, we examined the relationship between trials to criterion and choice latency. If mice with high latencies show low trials to criterion, this result may indicate a speed-accuracy trade-off strategy. Although not significant, mean correct latency exhibited a negative correlation with trials to criterion on ED shift performance $(r=-.25, p=.15)$. The relationship between ED trials to criterion and mean correct latencies was also examined, using only the mice in the highest and lowest quartiles of trials to criterion in the ED phase, as has been described previously for other measures (Swerdlow et al., 2006). The mean correct latencies of young mice with high versus low ED trials to criterion did not differ $[F(1,8)=3.0, p=.21$; Figure $5 \mathrm{~A}]$. Old mice with low ED trials to criterion were significantly slower than old mice with high ED trials to criterion $[F(1,8)=6.4$, $p<.05$; Figure 5B].

\section{DISCUSSION}

In the present study, we demonstrated that mice can readily be trained to discriminate between odors or platforms in performance on the ASST. Surprisingly, however, aged (24 months) mice did not exhibit a deficit in ASST performance, as compared with young (4 months) mice, as measured by trials to criterion. Aged mice did, however, exhibit significantly longer correct latencies than did young mice, specifically in the more difficult stage of the task - that is, the ED shift stage - with no differences observed at any other stage. This slowing of performance in some mice may reflect a speed-accuracy trade-off. This interpretation is supported by our finding that the aged mice with the lowest trials to criterion exhibited the longest choice latencies, which was not observed in the young mice. These data support the conclusion that aged mice may use a different strategy (speed-accuracy tradeoff) in the ASST to maintain accurate performance.

The validity of the ASST for probing executive functions is thought to be dependent on observing a significant increase in trials to criterion for the ED stage, as compared with the ID stage. Here, we observed a clear ID/ED difference irrespective of initial perceptual dimension, suggesting that ED shift performance assessed attentional set shifting. This ID/ED shift has not always been observed with mice in this task, likely due to methodological differences. To our knowledge, only two (DeSteno \& Schmauss, 2008; Papaleo et al., 2008) of eight (Brigman, Bussey, Saksida, \& Rothblat, 2005; Colacicco, Welzl, Lipp, \& Wurbel, 2002; Garner, Thogerson, Wurbel, Murray, \& Mench, 2006; Laurent \& Podhorna, 2004; Levi, Kofman, Schwebel, \& Shaldubina, 2008; Zhuo et al., 2007) mouse studies have demonstrated an ID/ED shift in parameters 
A

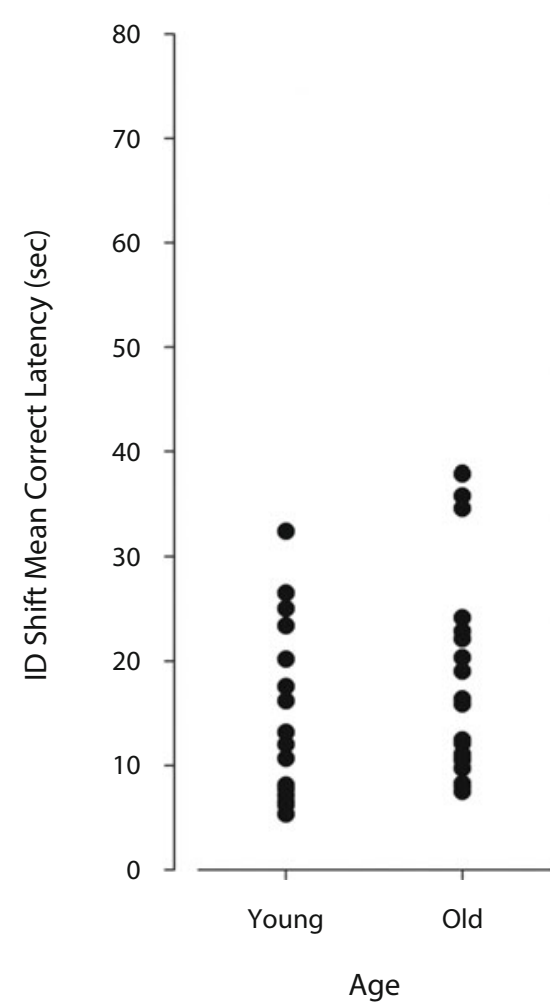

B

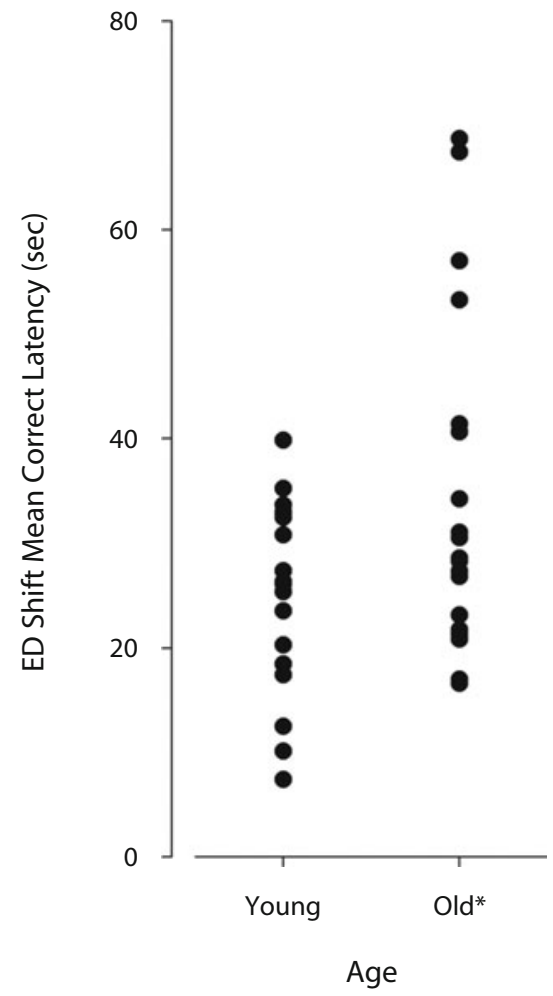

Figure 4. Individual mean correct latencies for mice at intradimensional (ID) and extradimensional (ED) shifts. The mean correct latencies of individual mice at the ID and ED shift stages were plotted and compared. (A) Latencies to perform ID shifts did not differ by age. (B) Latencies to perform ED shifts did, however, separate by age, with some old mice (age unimpaired) exhibiting performance at levels comparable to those of young mice, whereas other mice exhibited an age-related decline in performance (age impaired) as measured by increased mean correct latency. $\quad * p<.05$ for test of equality of error variance, as compared with young mice.

consistent with rats and humans (reviewed in Young, Powell, et al., 2009). Bisonette et al. (2008) utilized four ID shift stages to ensure that mice showed an attentional set when moved to the ED stage. The use of multiple ID stages to demonstrate an ID/ED effect is in contrast to the present study, in which only one ID shift was utilized, consistent with the original report for the task in rats (Birrell \& Brown, 2000). The data presented here support the conclusion that mice can form an attentional set, with ID/ ED differences observed in trials to criterion and mean correct latency. The present data also extend the dimensions from which stimuli can be selected when ASST is assessed, since the mice readily utilized platform cues to discriminate between stimuli. These findings are consistent with the utility of platforms as cues for rats in the cross-maze set-shifting paradigm (Stefani et al., 2003; Stefani \& Moghaddam, 2003).

Only one aging study (Zhuo et al., 2007) in mice in the ASST has been reported previously. This longitudinal study assessed the ASST performance of mice with the amyloid precursor protein mutation (TG2576). Although an effect of aging was observed for every stage in the task, neither ID/ED shifts nor performance differences from simple to reversal learning were observed (Zhuo et al., 2007). Thus, although an effect of aging was observed, the interpretation of the data remains ambiguous, since there was not a clear attentional set formed in these mice. These ambiguities also make it more difficult to compare with the present study. The present data do not fully support the idea that mice exhibit age-related deficits in performance of a set-shifting task. This finding is in contrast with previous human (Ashendorf \& McCaffrey, 2008; Owen et al., 1991; Rhodes, 2004) and rat (Barense et al., 2002) studies and may reflect the fact that the mice in the present study were younger than is required for an observed deficit in trials to criterion. The data do support the conclusion, however, that some aged mice did begin to exhibit altered performance in the task, because we found much higher variability in latency to respond in aged mice than in young mice. Although humans and rats exhibit poorer trials to criterion in ED shifting (Barense et al., 2002; Owen et al., 1991), aged mice in the present study exhibited longer mean correct latencies in ED shifting. The strategy for maintenance of accuracy at the ex- 
A

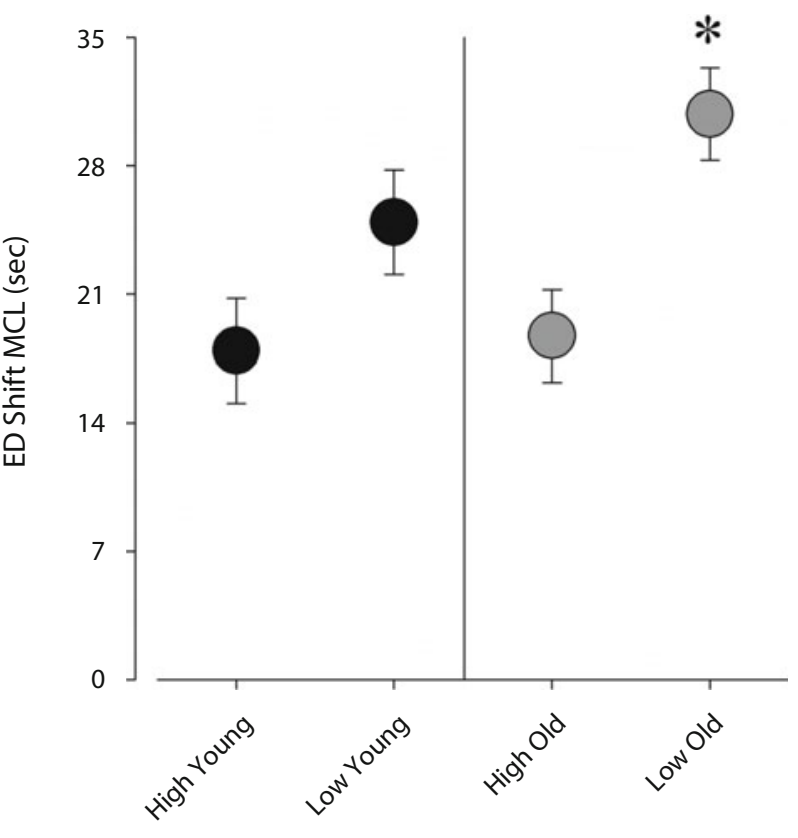

ED Shift Trials by Quartile
Figure 5. Examination of the relationship between extradimensional (ED) shift trials to criterion and mean correct latencies for young and old mice. The ED shift performance of old and young mice, as measured by trials to criterion, was split within the two age groups into high (poor performers) and low (good performers) quartiles (i.e., top and bottom $25 \%$ ). The mean correct latencies (MCLs) of these mice were then compared between quartiles within each age group. (A) Top performing young mice, as measured by ED trials to criterion, did not differ significantly in speed from poorer performing mice. (B) Older mice that performed well on ED shift trials to criterion were significantly slower than poorly performing older mice. Data are presented as mean $\pm S E M .{ }^{*} p<$ .05 when compared with poor performing old mice.

pense of speed is consistently observed in psychological testing in humans, including the elderly, and is referred to as a speed-accuracy trade-off (Busemeyer \& Townsend, 1993). The observation that the aged mice that performed most accurately in this test were significantly slower than poorer performing older mice supports the hypothesis that they adopted a speed-accuracy trade-off strategy. Younger mice, however, did not show a relationship between mean correct latency and accuracy, suggesting that this strategy was adopted only in the aged group.

Previous studies have suggested that mice demonstrate a speed-accuracy trade-off in other cognitive domains, especially when olfactory cues are used. In the odor discrimination choice reaction time task, increasing odor similarity, which presumably increases discrimination difficulty, resulted in longer reaction times in mice that maintained high accuracy (Abraham et al., 2004; Rinberg et al., 2006). Interestingly, when challenged similarly, rats did not lengthen their reaction times but committed more errors, reducing their accuracy in responding (Uchida \& Mainen, 2003). Therefore, consistent with these findings, increased task difficulty with ED rule shifting (Figure 2) and age in mice resulted in longer latencies (Figures 3 and 5), whereas rats were reported to exhibit an increase in errors (Barense et al., 2002). When performing a sustained attention task, aged rats exhibited both reduced accuracy and longer response latencies, when compared with younger rats (Muir, Fischer, \& Björklund, 1999). These results were interpreted as indicative of age-induced inattention, although processing speed deficiencies may have contributed as performance improved to levels comparable to those for younger rats with a small increase in stimulus duration (Muir et al., 1999). Thus, caution must be taken when interpreting data that may have been confounded by processing speed differences in rodents. These data highlight the utility of the speed-accuracy trade-off as a strategy in older animals for maintaining accuracy of performance. These speed-accuracy trade-off data in olfactory discrimination therefore support the hypothesis that older mice may have had greater difficulty in performing the ED shift, as compared with younger mice. It is certainly possible that latency increases were due to nonselective slowing of locomotor activity in aged mice; however, the restriction of these findings to stages that were most difficult (i.e., latency reductions were observed only in the ED stage, and not in the ID stage) would argue against this interpretation. In addition, the aged mice in these studies did not show a significant change in locomotor activity/exploration, as measured in the open field via distance traveled or average velocity (data not shown), suggesting that the long response rate was not due simply to nonspecific reductions in motor activation.

In the present study, young and old mice did not differ in simple discrimination learning (SD, CD, and ID shifting), as measured by trials to criterion or mean correct latency. Although there was an effect of stage, this effect was observed only as the mean correct latency for CD being shorter than that for SD or ID, likely due to the familiarity of the stimuli. The reversal learning performances of the mice (CDR, IDR, and EDR shifts) were significantly poorer, as measured by trials to criterion, than those for simple learning stages (CD, ID, ED shifts), however. These data indicate that the mice had acquired the discrimination rules and were not simply using an unknown strategy (e.g., bait scent) to perform the task. No effect of age on reversal learning performance, as measured by either errors or response latencies, was observed, findings that are consistent with the results of human studies of aging with similar tasks (Owen et al., 1991). Therefore, in the present study, the only age-related effect on mouse performance on the ASST appeared to be selective for response latencies in ED shifting. The selectivity for aging effects on this stage exhibits some consistency with the findings of human and rat studies (Barense et al., 2002; Owen et al., 1991). Another practical consideration of the use of the ASST in modeling cognitive decline with age is whether or not there is enough power to detect individual differences across aged animals for future mechanistic or treatment studies. Although power to detect AI and AU groups will vary with the criterion used (e.g., median 
split, quartile extremes, or two standard deviations from the mean of young performers) and with the method (e.g., age at testing), in the present study, aged animals showed the highest variability in ED latency performance (Figure 4B). Thus, this measure may allow for the strongest detection of differences in individual task performance, as compared with other phases of the task, or in trials to criterion measures.

Although the relationship between speed and accuracy is not always clear, there is evidence to support a speedaccuracy effect in ED shifting in mice performing the ASST. Bissonette et al. (2008) demonstrated that, consistent with human and rat neuroanatomical studies (Birrell \& Brown, 2000; McAlonan \& Brown, 2003; Owen et al., 1991), medial prefrontal cortex (mPFC) lesioned mice exhibited impaired ED shifts, whereas orbitofrontal lesioned mice exhibited impaired reversal learning. Interestingly, mice with mPFC lesions exhibited a poorer accuracy in the ED shifting stage only, whereas the response latencies of these mice were shorter than those of sham lesioned controls in ED shifting only (Bissonette et al., 2008), suggesting that faster responses may be linked to poor performance in impaired animals. The present findings suggest that the converse may also be observed, with age-impaired animals lengthening response time to maintain accuracy. In conclusion, mice can demonstrate an attentional set shift. Moreover, platforms can be used as a perceptual dimension, which may alleviate the ambiguity of differentiating between digging and sampling when digging media are used. The present study adds to the cross-species translational validity of using the ASST in mice and highlights the importance of assessing latency to respond, because this measure may unmask subtle cognitive differences or strategies. Finally, the ASST in mice may be useful for examining compensatory changes or resilience factors (Mhyre et al., 2005), which may contribute to successful cognitive aging (Glatt et al., 2007) in the executive function domain.

\section{AUTHOR NOTE}

This study was supported, in part, by a Sam and Rose Stein Institute for Research on Aging Fellowship (J.W.Y.) and a junior faculty pilot award (V.B.R.), as well as by the Veterans Affairs Center of Excellence for Stress and Mental Health, and by National Institute of Mental Health Grants P30 MH080002-01 and R01 MH071916. We thank Verity Brown and her laboratory for advice on statistical analyses and the setup of the ASST. We also thank Christine Scott and Mahalah Buell for their assistance. All of the authors report no conflict of interest. Correspondence concerning this article should be addressed to V. B. Risbrough, Department of Psychiatry, University of California, San Diego, 9500 Gilman Drive MC 0804, La Jolla, CA 92093-0804 (e-mail: vrisbrough@ucsd.edu).

\section{REFERENCES}

Abraham, N. M., Spors, H., Carleton, A., Margrie, T. W., Kuner, T., \& SCHAEFER, A. T. (2004). Maintaining accuracy at the expense of speed: Stimulus similarity defines odor discrimination time in mice. Neuron, 44, 865-876.

Anstey, K. J., \& Low, L. F. (2004). Normal cognitive changes in aging. Australian Family Physician, 33, 783-787.

Ashendorf, L., \& McCAFFrey, R. J. (2008). Exploring age-related decline on the Wisconsin Card Sorting Test. Clinical Neuropsychologist, 22, 262-272.
Barense, M. D., Fox, M. T., \& Baxter, M. G. (2002). Aged rats are impaired on an attentional set-shifting task sensitive to medial frontal cortex damage in young rats. Learning \& Memory, 9, 191-201.

BirRell, J. M., \& BRown, V. J. (2000). Medial frontal cortex mediates perceptual attentional set shifting in the rat. Journal of Neuroscience, 20, 4320-4324.

Bissonette, G. B., Martins, G. J., Franz, T. M., Harper, E. S., Schoenbaum, G., \& Powell, E. M. (2008). Double dissociation of the effects of medial and orbital prefrontal cortical lesions on attentional and affective shifts in mice. Journal of Neuroscience, 28, 11124-11130.

Brigman, J. L., Bussey, T. J., Saksida, L. M., \& Rothblat, L. A. (2005). Discrimination of multidimensional visual stimuli by mice: Intra- and extradimensional shifts. Behavioral Neuroscience, 119, 839-842.

Busemeyer, J. R., \& Townsend, J. T. (1993). Decision field theory: A dynamic-cognitive approach to decision making in an uncertain environment. Psychological Review, 100, 432-459.

Caine, S. B., Negus, S. S., \& Mello, N. K. (1999). Method for training operant responding and evaluating cocaine self-administration behavior in mutant mice. Psychopharmacology, 147, 22-24.

Colacicco, G., Welzl, H., Lipp, H. P., \& Wurbel, H. (2002). Attentional set-shifting in mice: Modification of a rat paradigm, and evidence for strain-dependent variation. Behavioural Brain Research, 132, 95-102.

DeSteno, D. A., \& Schmauss, C. (2008). Induction of early growth response gene 2 expression in the forebrain of mice performing an attention-set-shifting task. Neuroscience, 152, 417-428.

Eling, P., Derckx, K., \& Maes, R. (2008). On the historical and conceptual background of the Wisconsin Card Sorting Test. Brain \& Cognition, 67, 247-253.

Garner, J. P., Thogerson, C. M., Wurbel, H., Murray, J. D., \& Mench, J. A. (2006). Animal neuropsychology: Validation of the intra-dimensional extra-dimensional set shifting task for mice. Behavioural Brain Research, 173, 53-61.

Gazzaley, A., \& D'Esposito, M. (2007). Top-down modulation and normal aging. In M. J. de Leon, D. A. Snider, \& H. Federoff (Eds.), Imaging and the aging brain (Annals of the New York Academy of Sciences, Vol. 1097, pp. 67-83). New York: New York Academy of Sciences.

Glatt, S. J., Chayavichitsilp, P., Depp, C., Schork, N. J., \& Jeste, D. V. (2007). Successful aging: From phenotype to genotype. Biological Psychiatry, 62, 282-293.

Hendrie, H. C., Albert, M. S., Butters, M. A., Gao, S., Knopman, D. S., LAUNER, L. J., ET AL. (2006). The NIH cognitive and emotional health project report of the critical evaluation study committee. Alzheimer's \& Dementia, 2, 12-32.

Kremen, W. S., Eisen, S. A., Tsuang, M. T., \& Lyons, M. J. (2007). Is the Wisconsin Card Sorting Test a useful neurocognitive endophenotype? American Journal of Medical Genetics B, 144, 403-406.

Laurent, V., \& Podhorna, J. (2004). Subchronic phencyclidine treatment impairs performance of C57BL/6 mice in the attentional setshifting task. Behavioural Pharmacology, 15, 141-148.

Lee, H. K., Min, S. S., Gallagher, M., \& Kirkwood, A. (2005) NMDA receptor-independent long-term depression correlates with successful aging in rats. Nature Neuroscience, 8, 1657-1659.

Levi, Y., Kofman, O., Schwebel, M., \& Shaldubina, A. (2008). Discrimination and avoidance learning in adult mice following developmental exposure to diisopropylfluorophosphate. Pharmacology Biochemistry \& Behavior, 88, 438-445.

McAlonan, K., \& Brown, V. J. (2003). Orbital prefrontal cortex mediates reversal learning and not attentional set shifting in the rat. Behavioural Brain Research, 146, 97-103.

Mhyre, T. R., Chesler, E. J., Thiruchelvam, M., Lungu, C., CorySlechta, D. A., Fry, J. D., ET AL. (2005). Heritability, correlations and in silico mapping of locomotor behavior and neurochemistry in inbred strains of mice. Genes, Brain, \& Behavior, 4, 209-228.

Muir, J. L., Fischer, W., \& BJöRKLund, A. (1999). Decline in visual attention and spatial memory in aged rats. Neurobiology of Aging, 20, 605-615.

Nicolle, M. M., \& BAXTER, M. G. (2003). Glutamate receptor binding in the frontal cortex and dorsal striatum of aged rats with impaired attentional set-shifting. European Journal of Neuroscience, 18, 3335-3342. 
Owen, A. M., Roberts, A. C., Polkey, C. E., Sahakian, B. J., \& RobBINS, T. W. (1991). Extra-dimensional versus intra-dimensional set shifting performance following frontal lobe excisions, temporal lobe excisions or amygdalo-hippocampectomy in man. Neuropsychologia, 29, 993-1006.

Papaleo, F., Crawley, J. N., Song, J., Lipska, B. K., Pickel, J., WeinBERGER, D. R., \& CHEN, J. (2008). Genetic dissection of the role of catechol-O-methyltransferase in cognition and stress reactivity in mice. Journal of Neuroscience, 28, 8709-8723.

Population Division, U.S. Census Bureau (2001). National population projections. Washington, DC: Author. Available: www.census .gov/population/www/projections/usinterimproj.

Rhodes, M. G. (2004). Age-related differences in performance on the Wisconsin card sorting test: A meta-analytic review. Psychology \& Aging, 19, 482-494.

Rinberg, D., Koulakov, A., \& Gelperin, A. (2006). Speed-accuracy tradeoff in olfaction. Neuron, 51, 351-358.

RowE, J. W., \& KAHN, R. L. (1987). Human aging: Usual and successful. Science, 237, 143-149.

Silverman, J. M., Schnaider-Beeri, M., Grossman, H. T., SchmeidLER, J., WANG, J. Y., \& LALlY, R. C. (2008). A phenotype for genetic studies of successful cognitive aging. American Journal of Medical Genetics B, 147, 167-173.

Stefani, M. R., Groth, K., \& Moghaddam, B. (2003). Glutamate receptors in the rat medial prefrontal cortex regulate set-shifting ability. Behavioral Neuroscience, 117, 728-737.

Stefani, M. R., \& Moghaddam, B. (2003). Distinct contributions of glutamate receptor subtypes to cognitive set-shifting abilities in the rat. In B. Moghaddam \& M. E. Wolf (Eds.), Glutamate and disorders of cognition and motivation (Annals of the New York Academy of Sciences, Vol. 1003, pp. 464-467). New York: New York Academy of Sciences.

Swerdlow, N. R., Light, G. A., Cadenhead, K. S., Sprock, J., Hsieh, M. H., \& BRAFF, D. L. (2006). Startle gating deficits in a large cohort of patients with schizophrenia: Relationship to medications, symptoms, neurocognition, and level of function. Archives of General Psychiatry, 63, 1325-1335.

UCHIDA, N., \& MAINEN, Z. F. (2003). Speed and accuracy of olfactory discrimination in the rat. Nature Neuroscience, 6, 1224-1229.
Velanova, K., Lustig, C., Jacoby, L. L., \& Buckner, R. L. (2007). Evidence for frontally mediated controlled processing differences in older adults. Cerebral Cortex, 17, 1033-1046.

Whalley, L. J., Deary, I. J., Appleton, C. L., \& Starr, J. M. (2004). Cognitive reserve and the neurobiology of cognitive aging. Ageing Research Reviews, 3, 369-382.

Young, J. W., Crawford, N., Kelly, J. S., Kerr, L. E., Marston, H. M., Spratt, C., ET AL. (2007). Impaired attention is central to the cognitive deficits observed in alpha 7 deficient mice. European Neuropsychopharmacology, 17, 145-155.

Young, J. W., Kerr, L. E., Kelly, J. S., Marston, H. M., Spratt, C., Finlayson, K., \& Sharkey, J. (2007). The odour span task: A novel paradigm for assessing working memory in mice. Neuropharmacology, 52, 634-645.

Young, J. W., Powell, S. B., Risbrough, V., Marston, H. M., \& GeYER, M. A. (2009). Using the MATRICS to guide development of a preclinical cognitive test battery for research in schizophrenia. Pharmacology \& Therapeutics, 122, 150-202.

Young, J. W., Sharkey, J., \& Finlayson, K. (2009). Progressive impairment in olfactory working memory in a mouse model of mild cognitive impairment. Neurobiology of Aging, 30, 1430-1443.

Zhang, H. Y., Watson, M. L., Gallagher, M., \& Nicolle, M. M. (2007). Muscarinic receptor-mediated GTP-Eu binding in the hippocampus and prefrontal cortex is correlated with spatial memory impairment in aged rats. Neurobiology of Aging, 28, 619-626.

Zhuo, J.-M., Prescott, S. L., Murray, M. E., Zhang, H.-Y., BaXter, M. G., \& Nicolle, M. M. (2007). Early discrimination reversal learning impairment and preserved spatial learning in a longitudinal study of Tg2576 APPsw mice. Neurobiology of Aging, 28, 1248-1257.

Zubenko, G. S., Stiffler, J. S., Hughes, H. B., III, Fatigati, M. J., \& Zubenko, W. N. (2002). Genome survey for loci that influence successful aging: Sample characterization, method validation, and initial results for the $\mathrm{Y}$ chromosome. American Journal of Geriatric Psychiatry, 10, 619-630.

(Manuscript received August 12, 2009; revision accepted for publication October 2, 2009.) 